\title{
Demo: Formalization IDEs Integrated with a Verifying Compiler
}

\author{
Daniel Welch \\ School of Computing, Clemson University \\ Clemson, SC 29634, USA \\ dtwelch@clemson.edu \\ Mike Kabbani \\ School of Computing, Clemson University \\ Clemson, SC 29634, USA \\ nkabban@clemson.edu
}

\begin{abstract}
This demonstration will illustrate both a web- and desktop-based formalization IDE (F-IDE) that are backed by a verifying compiler for the RESOLVE specification and programming language. Each IDE we demo supports construction of mathematical developments, formal interface specifications of generic, object-based concepts, and alternative implementations annotated with internal assertions to enable verification. While the first portion of the demo will illustrate the language and verification in the context of the webbased environment, the second half will demonstrate features of a newer desktop-based IDE that provides additional modern IDE amenities beyond those offered by the web-based version. Each IDE we present integrates feedback for mathematical and programmatic type checking, proving, among others, and permit users to generate and run executable, property-preserving Java.
\end{abstract}

\section{Introduction}

The F-IDEs we present facilitate construction of verified components and component-based software in RESOLVE, an integrated specification and programming language designed to support construction and modular verification of object-based software [12] In particular, the newer developmental desktop-based F-IDE we present integrates support for RESOLVE into commercial and open source JetBrains IDEs [6] by leveraging a combination of features from related efforts discussed in section 2 .

Figure 1 highlights several of the amenities provided by the desktop-based version such as contextual completion suggestions for partially typed references appearing in specifications (and code), as well as language keywords. As expected of modern environments, the F-IDE provides responsive contextual completion suggestions for partially typed keywords and references (illustrated in Figure 1). Such completions are performed in an intelligent way: That is, the IDE only suggests keywords in regions where they are grammatically valid, and avoids offering completions for abstract mathematical definitions in programmatic contexts (e.g., statement lists) where references to strictly programmatic variables and operations are expected.

Each of the F-IDEs we present is supported by an in-development verifying compiler $[4,13]$, which allows learners to verify their code in a 'push-button' manner (Figure 3, left), then, from this verified

Permission to make digital or hard copies of part or all of this work for personal or classroom use is granted without fee provided that copies are not made or distributed for profit or commercial advantage and that copies bear this notice and the full citation on the first page. Copyrights for third-party components of this work must be honored. For all other uses, contact the owner/author(s).

ICOOOLPS'17, Barcelona, Spain

(c) 2017 Copyright held by the owner/author(s). 978-1-4503-5088-4/17/06 .. $\$ 15.00$ DOI: $10.1145 / 3098572.3098580$

\author{
Blair Durkee
School of Computing, Clemson University \\ Blair Durkee
School of Computing, Clemson University \\ Clemson, SC 29634, USA \\ bdurkee@clemson.edu \\ Murali Sitaraman \\ School of Computing, Clemson University \\ Clemson, SC 29634, USA \\ msitara@clemson.edu

Murali Sitaraman
School of Computing, Clemson University
Clemson, SC 29634, USA

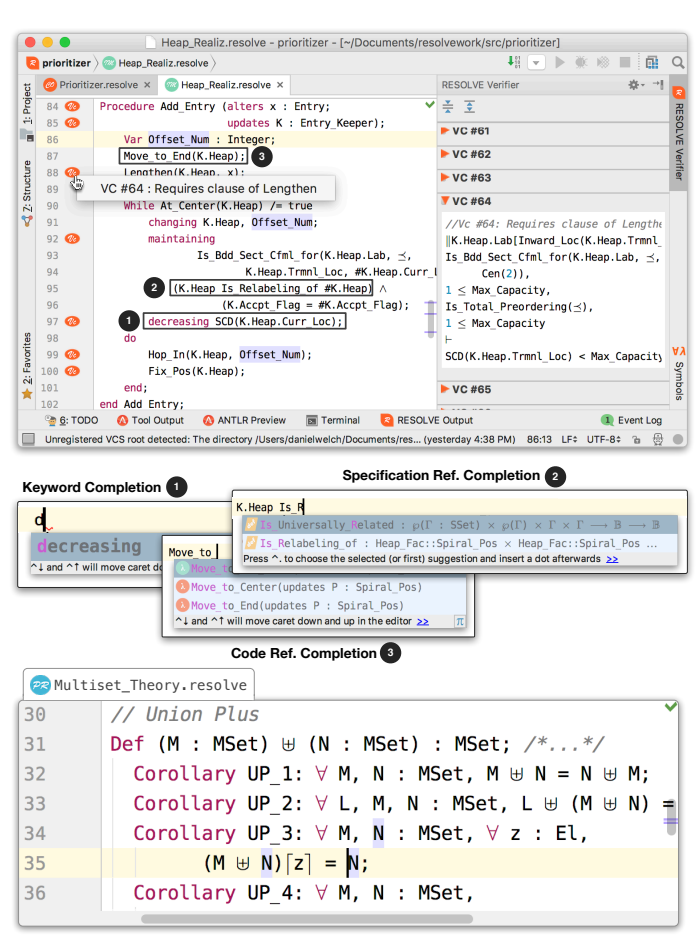

Figure 1. RESOLVE JetBrains IDE integration; code and specification editing features.

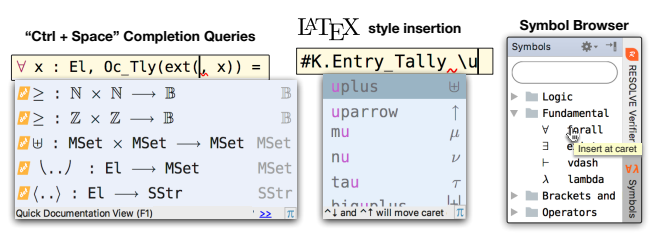

Figure 2. Specification editing features.

code, generate property preserving, executable Java-and run it with the click of a button (Figure 3, right, bottom). More than simplifying interaction with the compiler, the environment also provides assistance with carrying out Design by Contract (DbC). This is done through tooltips that not only show pre- and post conditions at the site of calls and module instantiations, but also displays (while typing) the formal parameter mode associated with each argument as it's being passed. 

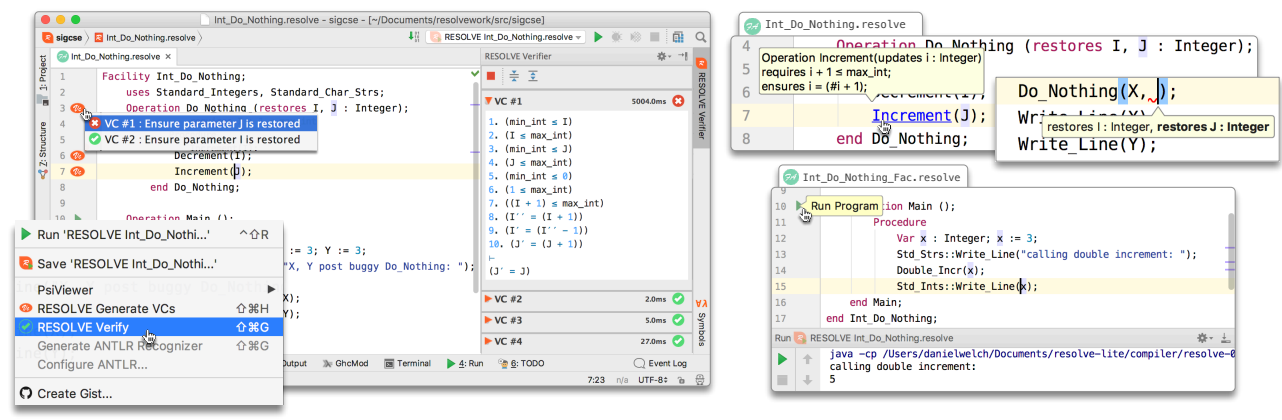

Figure 3. RESOLVE's in-house automated prover (left), DbC assistance (right, top), and code gen (right, bottom).

\subsection{Mathematical Assertions and Type Checking}

Since mathematical assertions and code coexist in close quarters within software components designed to be amenable to verification, to help underscore the language's enforced separation between formal mathematical specification and executable code, RESOLVE makes extensive use of non-ASCII unicode symbols throughout specifications. The newer, desktop-based F-IDE in particular allows users to easily insert these special characters into the active editor via LTEX-style insertion commands (Figure 2-middle, right) or by 'pinging' the scope for available operators (Figure 2-left).

The importance of mathematical annotations is also what distinguishes the type checking and help available in the RESOLVE F-IDE from that in typical object-based languages. The mathematical type checking of assertions is done in such a way that decouples the type checking problem from the verification problem. Specification languages in which assertions are expressed, strictly speaking, do not need type systems, since the code is never executed. No operations are performed on data because the very notion of performing operations is outside the language's scope. Rather, specifications state logical expressions that can be used by a prover to verify the correctness of software. Among the benefits of type checking of mathematical annotations is to shorten the learning curve for programmers who are new to specification languages and verification in general. By including a type system the exhibits the same expected behavior as implementation languages, the barrier to entry is somewhat lowered. The type checker allows the programmer to ensure a certain level of integrity of their specifications without the need for invoking the prover. If a specification is obviously wrong, such as using a length-of operator on a scalar value, then the type checker will raise an error before the prover engages. This can be especially beneficial when the prover is long-running or requires additional user interaction. A technical benefit is the ability to offload complexity from the prover. Using a type checker enables the prover to make certain basic assumptions about incoming verification conditions. Fewer theorems and inferences will then be required to dispatch these VCs.

\subsection{A Limited Example}

A comprehensive component-based case study built using the IDE is the topic of [16]. The bottom half of Figure 1 shows a snippet of Multiset_Theory which houses a selection of mathematical notations and operators that can be used to formalize component specifications, as well as a selection of theorems and corollaries required for automated proof. The RESOLVE compiler type-checks such formal annotations to ensure that specifications are well-formed-thus keeping the prover from needing to interpret nonsensical assertions. And since the compiler is fully integrated, the F-IDE dynamically annotates such errors in the editor, which provides users with a quick feedback loop on the specifications as they are being written.

The top half on the figure illustrates the implementation-level code involving a loop. The behavior of the loop in this particular implementation is captured through several formal annotations including a maintaining clause (i.e., a loop invariant) that must hold at the beginning and end of each iteration and a decreasing clause (i.e., a progress metric) for proving termination.

This figure also illustrates how the F-IDE presents proof obligations (or, verification conditions) to users. Lines that generate one or more proof obligations are marked by a (2) icon in the gutter and can be selected and viewed in the "RESOLVE Verifier" panel.

While RESOLVE has been used extensively in software engineering education in conjunction with the web-based version of the IDE [2, 3], the plan is to use the newer, desktop based F-IDE discussed here in the classrooms in the Fall of 2017.

\section{Related Work}

In this section we discuss only a handful of efforts most closely related to our approach: namely those that (1) tackle full functional verification of imperative, sequential programs and (2) enjoy prominent F-IDE support.

$\mathrm{KeY}$. The first effort we discuss is $\mathrm{KeY}[1]-$ a deductive verification tool for code written in Java. KeY leverages the Java Modeling Language (JML) [8] for expressing formal specifications and-for program verification-uses its own integrated backend prover. In terms of F-IDE support, KeY provides two systems. The first is a standalone Java application that serves as the standard frontend for the tool, simply called KeY GUI. This environment functions as a general purpose KeY editor that allows users to perform functional verification of JML-annotated programs, explore resultant proof obligations, and in general interact with the prover in a manner reminiscent of proof assistants such as Coq [14]. The second frontend is an extension that integrates KeY into Eclipse [5]. Upon writing JML-annotated code, or changing existing code, the plugin automatically invokes KeY's prover in the background (e.g., upon saving the document), and marks methods within the editor accordingly depending on whether or not the corresponding proof succeeded. The F-IDE tracks these annotations persistently across runs, visually indicating dependencies not yet fully verified. 
Dafny. The second effort is Dafny [9], an object-oriented, imperative language which (like RESOLVE) is designed for formal specification and automated verification. As a result, the language includes syntactic slots for formal annotations and leverages Microsoft's Z3 on the backend for automated verification. F-IDE support for Dafny is provided through an extension to Microsoft Visual Studio [10]. Features include extensive background caching (so proofs don't have to be recomputed unnecessarily) as well as a verification debugger that permits users to explore the states leading to a failed (or, unproven) assertion.

Eiffel. Eiffel, the language that pioneered Design by Contract (DbC), provides F-IDE support through EiffelStudio-an environment that permits the construction of formally specified Eiffel software and (among many other features) the ability to automatically generate test cases from the formal specifications. Due to the language's emphasis on safety and specification, Eiffel has been used as a language-frontend for multiple third party verification efforts such as [15]-as well as the even more recent, impressive feat comprising the specification and verification of Eiffel's primary container library: EiffelBase2 [11].

Each of the IDEs we've built to support RESOLVE (including the newer, desktop-based version) diverge from the above efforts largely in terms of user feedback and process. For example, verification in RESOLVE is viewed largely as an iterative 'debugging' process, wherein users press the verify button and receive notification from the verifier as to which proof obligations were successfully proven (and which weren't)-resulting in the unverified obligations being overlaid onto the relevant lines for further examination. And while proof obligations in RESOLVE themselves are human readable, and therefore differ substantially from those generated by the Boogie proof obligation generator, which, in general, are not designed to be read by users ${ }^{1}, \mathrm{KeY}$ uses an altogether different approach that permits users to complete a failed verification attempt by hand using a proof-assistant style of interaction.

\section{Conclusions and Demo Outline}

This demo abstract summarizes characteristics of the RESOLVE language and features of its two IDE frontends. The first half of the demo will comprise of an overview of the web-based IDE as well as an overview of the RESOLVE language, and verification of a component-based example. In the demo we emphasize how the verification conditions are generated, their role in proving, and how the environment provides verification feedback to users. The second half of the demo will discuss features of the newer, desktopbased IDE and (time permitting) future envisioned additions to the desktop-based environment.

\section{Acknowledgments}

This research is funded by US NSF grants CCF-1161916 and DUE1611714.

\section{References}

[1] Wolfgang Ahrendt, Bernhard Beckert, Richard Bubel, Reiner Hähnle, Peter H. Schmitt, and Mattias Ulbrich. 2016. Deductive Software Verification - The KeY Book - From Theory to Practice. Lecture Notes in Computer Science, Vol. 10001 Springer, Cham, Switzerland.

\footnotetext{
${ }^{1}$ Although this is remedied in part when used in conjunction with the Boogie Verification Debugger (BVD) tool [7].
}

[2] C. T. Cook, H. Harton, H. Smith, and M. Sitaraman. 2012. Specification engineering and modular verification using a web-integrated verifying compiler. In ICSE 2012. 1379-1382. DOI : https://doi.org/10.1109/ICSE.2012.6227243

[3] Svetlana V. Drachova, Jason O. Hallstrom, Joseph E. Hollingsworth, Joan Krone, Richard Pak, and Murali Sitaraman. 2015. Teaching Mathematical Reasoning Principles for Software Correctness and Its Assessment. TOCE 15, 3 (2015), 15:1-15:22. DOI : https://doi.org/10.1145/2716316

[4] Heather Harton. 2011. Mechanical and Modular Verification Condition Generation For Object-Based Software. Ph.D. Dissertation. Clemson University.

[5] Martin Hentschel, Stefan Käsdorf, Reiner Hähnle, and Richard Bubel. 2014. An Interactive Verification Tool Meets an IDE. In IFM 2014 (LNCS), Elvira Albert and Emil Sekerinski (Eds.), Vol. 8739. Springer, Cham, Switzerland, 55-70. DOI : https://doi.org/10.1007/978-3-319-10181-1_4

[6] JetBrains. 2017. IDEs. Software, https://www.jetbrains.com/. (2017).

[7] Claire Le Goues, K. Rustan M. Leino, and Micha 1 Moskal. 2011. The Boogie Verification Debugger (Tool Paper). In SEFM 2011 (LNCS), Gilles Barthe, Alberto Pardo, and Gerardo Schneider (Eds.). Springer Berlin Heidelberg, Berlin, Heidelberg, 407-414.

[8] Gary T. Leavens, Erik Poll, Curtis Clifton, Yoonsik Cheon, Clyde Ruby, David Cok, Peter Müller, Joseph Kiniry, Patrice Chalin, Daniel M. Zimmerman, and Werner Dietl. 2013. 7ML Reference Manual. Revision 2344.

[9] K. Rustan M. Leino. 2010. Dafny: An Automatic Program Verifier for Functional Correctness. In LPAR 2010 (LNCS), Edmund M. Clarke and Andrei Voronkov (Eds.), Vol. 6355. Springer, Heidelberg, Berlin, Heidelberg, 348-370. DOI : https: //doi.org/10.1007/978-3-642-17511-4_20

[10] K. Rustan M. Leino and Philipp Rümmer. 2014. The Dafny Integrated Development Environment. In F-IDE 2014 (EPTCS), Catherine Dubois, Dimitra Giannakopoulou, and Dominique Méry (Eds.), Vol. 149. EPTCS, 3-15. DOI: https://doi.org/10.4204/EPTCS.149

[11] Nadia Polikarpova, Julian Tschannen, and Carlo A. Furia. 2015. A Fully Verified Container Library. Springer, Cham, 414-434. DOI : https://doi.org/10.1007/ 978-3-319-19249-9_26

[12] Murali Sitaraman, Bruce Adcock, Jeremy Avigad, Derek Bronish, Paolo Bucci, David Frazier, Harvey M. Friedman, Heather Harton, Wayne Heym, Jason Kirschenbaum, Joan Krone, Hampton Smith, and Bruce W. Weide. 2011. Building a Push-button RESOLVE Verifier: Progress and Challenges. Formal Aspects of Computing. 23, 5 (sep 2011), 607-626. DOI: https://doi.org/10.1007/ s00165-010-0154-3

[13] Hampton Smith. 2013. Engineering Specifications and Mathematics for Verified Software. Ph.D. Dissertation. Clemson University.

[14] The Coq Development Team. 2016. The Coq Proof Assistant Reference Manual. V8.6.

[15] Julian Tschannen, Carlo A. Furia, Martin Nordio, and Bertrand Meyer. 2011. Verifying Eiffel Programs with Boogie. In First International Workshop on Intermediate Verification Languages, BOOGIE (2011). http://arxiv.org/abs/1106.4700

[16] Daniel Welch and Murali Sitaraman. 2017. Engineering and Employing Reusable Software Components for Modular Verification. In ICSR 2017 (In publication) (LNCS), Goetz Botterweck and Claudia Werner (Eds.), Vol. 10221. Springer International Publishing, Cham, Heidelberg, 139-154. DOI : https://doi.org/10.1007/ 978-3-319-56856-0 